\title{
Personality Construct Similarity in Israel and the United States
}

\author{
1. Montag \\ The Medical Instutute of Road Safery and Tel Aviv University. Israel \\ And:ew L. Comrey \\ University of California, Los Angelos
}

\begin{abstract}
A translated version of the Comrey Personality Scales was administered to 185 male applicants for motor vehicle operator's license in Israel after special measures were taken to reduce response distor. tion. Total scores were computed for these subjects over each of the 40 homogeneous item subgroups that define the eight personality factors in the tax. onomy underlying the Comrey Personality Scales. Factor analysis of the intercorrelations among these 40 subvariables resulted in substantial confirmation of seven of the eight hypothesized factors. Failure of the eighth factor to emerge may have been due to higher average scores and lower standard deviations for the item groups defining this particular factor, possibly caused by the special conditions under which the subjects were tested. The overall results of this study provide further evidence confirming the robustness of the taxonomy of personality traits underlying the Comrey Personality Scales across language and cultural boundaries.
\end{abstract}

Individuals seeking a public vehicle driving license in the State of Israel are required to undergo full medical and psychological examination to determine their fitness. Individuals seeking a private vehicle operator's license are also required to pass the same screening provided they have had an accident, unsatisfactory military record, rejection from military service, or other indication of their possible lack of fitness for driving. One of the psychological tests that APPLIED PSYCHOLOGICAL MEASUREMENT Vol. 6, No. 1, Winter 1982, pp. 61-67

(C) Copyright 1982 Applied Psychological Measurement Inc. 0146-6216/82/010061-07\$1.35 has been employed as a possible component of the ultimate screening battery is a translated version of the Comrey Personality Scales (Comrey, 1970). Considerable evidence has been accumulated pointing to the possible value of this instrument for purposes of psychological screening (Comrey, 1980), leading to consideration of the hypothesis that it might be useful for screening driving license applicants in Israel.

The Comrey Personality Scales are based on a personality taxonomy developed over a long series of research investigations. The personality constructs making up this system are the follow. ing: Trust vs. Defensiveness (T), Orderliness vs. Lack of Compulsion (O), Social Conformity vs. Rebelliousness (C), Activity vs. Lack of Energy (A), Emotional Stability vs. Neuroticism (\$), Extraversion vs. Introversion (E), Masculinity vs. Femininity (M), and Empathy vs. Egocentrism (P). This personality taxonomy, as developed by Comrey and coauthors, building on the work of other factor theorists, has been substantially replicated by other investigators using different samples of subjects in the United States (Vandenberg \& Price, 1978) and in other countries (Forbes, Dexter, \& Comrey, 1974; Rodrigues \& Comrey, 1974).

The purpose of the present investigation was to determine whether the basic personality taxonomy underlying the Comrey Personality Scales could be replicared in Israel using a version of this instrument translated into Hebrew. 
Since the Comrey Personality Scales have shown capabilities for detecting personality abnormallties in English-speaking subjects (Comrey, 1980), if the same basic factors appeared with Israelis, this instrument might have utility in ISrael for detecting psychological characteristics in driving applicants that might bear upon their suitability for licensing. To show that the same personality factors that have previously been found in other countries can be generated in an Israeli population is also of general scientific interest, quite apart from its specific utility for screening purposes in Israel.

\section{Method}

The Comrey Personality Scales were translated into Hebrew and administered to 185 male applicants for Israeli driving licenses, both private and public (fourteen female applicants were not used in this analysis, since they constituted too small a group to provide useful data). Other tests were also administered to this group, but these results are not reported here.

Since applicants wish to be licensed, they are motivated to do well; there is thus great temptation to falsify personality test responses in the context under which the data were collected. Comrey and Backer (1975) have pointed out that these kinds of testing conditions can markedly affect personality test responses on the Comrey Personality Scales and, hence, the underlying factor structure that can be derived from such data. The first author has developed a technique to substantially reduce the tendency of subjects to falsify their responses under these conditions (Montag, 1978). ${ }^{1}$ Prior to taking the personality tests of interest, subjects are given a test of 20 very transparent social desirability items. The scores on this pretest are given back to the examinees so they can be aware of the extent of their apparent "faking good" on the pretest.

'The first author's research with this method is also referred to in earlier publications by Eysenck, Eysenck, and Show (1974) and by Eysenck and Eysenck (1976, pp. 165-166, 200-201).
Following this, the tests of primary interest are administered, with the expectation that the examinees will show less tendency to falsify or to distort their responses in a socially desirable way in the hope of increasing their chances of being licensed. This procedure was followed in the present study prior to the administration of the Comrey Personality Scales. This step was regarded as essential because unpublished studies in the past using job applicant populations without such procedures to reduce falsification have resulted in a collapse of the basic factor structure underlying the Comrey Personality Scales.

\section{Analysing}

Each of the eight personality factors on the Comrey Personality Scales is measured by five subvariables, called factored homogeneous iiem dimensions (FHIDs). Each FHID, in tum, consists of four homogeneous items, two positively stated and two negatively stated with respect to the FHID name. Total scores were computed for each subject in this investigation for each of the 40 FHIDs by summing the item scores after reversing the negatively stated item scores. Since each item is scored on a seven-choice scale, the possible scores on a given FHID range from 4 to 28. These 40 FHID scores were intercorrelated by Pearson product-moment $r$ and subjected to factor analysis by the minimum residual method with varimax rotations (Comrey, 1973).

If the original factor structure reported in the Comrey Personality Scales test manual (Comrey, 1970) were to be replicated, there should be exactly eight factors, with the 40 FHID variables showing substantial loadings only on the particular factors they are supposed to define. In order not to prejudge the number of factors in the present data, 15 factors were extracted and examined for possible retention in the rotation process. Factors 10 through 14 had no loading as high as .3, and Factor 15 had only one such loading, so no rotational solution was carried out for more than nine factors. Trial varimax rotational solutions were carried out for six, seven, 
eight, and nine factors. The nine-factor and eight-factor solutions showed only seven major interpretable factors, whereas the six-factor solution collapsed two major factors into one factor. Accordingly, seven factors was concluded to be the proper number. Actually, the sums of squares of factor loadings showed a major gap between the sixth and seventh factors, implying the appropriateness of a six-factor solution, but this was rejected because of the persistent appearance of a seventh interpretable factor of substantial magnitude. No more than seven factors, however, could be retained.

After the decision was made to retain seven factors, a refactoring was carried out, iterating the seven principal factor solution communalities until stability was obtained, starting with the minimum residual solution communalities. These seven principal factors with stabilized communalities were rotated by varimaz to give the final solution. ${ }^{2}$

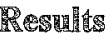

Each of the seven rotated factors is shown in Table 1 with loadings for those FHIDs that were expected to define these factors. For comparison purposes, the loadings obtained for these FHIDs in the original normative group analysis defining the Comrey Personality Scales taxonomy (Comrey, 1970) are also shown in Table 1. Loadings of .3 or more for FHIDs not expected to define the factors, if any, will be mentioned below in the discussion of each separate factor.

Trust vs. Defensiveness (T). High scores on this factor (Factor I) indicate a belief in the basic honesty, trustworthiness, and good intentions of other people. Loadings of .3 or more in the cur-

${ }^{2}$ See NAPS Document No. 03898 for five pages of supplementary material from NAPS, Microfiche Publications, P.O. Box 3513, Grand Central Station, New York NY 10163. This material includes the $40 \times 40$ matrix of correlations among FHIDs, the matrix of seven extracted factors, and the matrix of seven normal varimax rotated factors as Tables 4,5 , and 6 , respectively. Remit in advance $\$ 4.00$ for microfiche copy or $\$ 7.75$ for photocopy. Outside U.S. and Canada add postage of $\$ 4.50$ for photocopies or $\$ 1.50$ for microfiche. rent analysis for FHIDs that were not expected to define this factor were as follows: Acceptance of Social Order (No. 12), .58; Lack of Depression (No. 22), .36; and Mood Stability (No. 25), .39. The corresponding loadings in the normative sample for these FHIDs were $.17, .20$, and .09 , respectively. The only major unexpected loading here was that for Acceptance of Social Order (No. 12), .58; it is supposed to define Factor IV, Social Conformity vs. Rebelliousness (C), which failed to emerge as a separate factor in the current analysis. Otherwise, the major loadings for this factor (see Table 1) were in substantial agreement with expectations on the basis of the original normative group results. The factor of Trust vs. Defensiveness (T) is regarded as being confirmed in this analysis.

Orderliness vs. Lack of Compulsion (O). High scores on this factor (Factor $\mathbb{I I}$ ) are characteristic of careful, meticulous, orderly, and highly organized individuals. Loadings of .3 or more in the current analysis for FHIDs that were not expected to define this factor were as follows: Intolerance of Nonconformity (No. 13), .58; and Need for Approval (No. 15), .42. The corresponding loadings in the normative sample were .31 and .26 , respectively. Both FHID variables 13 and 15 were supposed to define Factor IV, Social Conformity vs. Rebelliousness $(\mathrm{C})$, which did not appear as a separate factor in the current amalysis. It is logical that major loadings for some of the FHIDs defining Factor IV should appear on Orderliness vs. Lack of Compulsion (O), since these two scales on the Comrey Personality Scales correlate .47 , higher than for any other two personality scales. Another of the Social Conformity FHIDs appeared with a major loading on the previous factor, Trust vs. Defensiveness (T). The correspondence between current and past loadings for Orderliness ws. Lack of Compulsion $(O)$ is sufficiently high to warrant the conclusion that this factor has been confirmed in the present analysis (see Table 1).

Social Conformity ws. Rebelliousness (C). Individuals with high scores accept the society as it is, resent nonconformity in others, seek the ap- 
Table 1

Current and Normative Loadings for FHIDs Defining Seven of Eight Expected Personal ity Factors

\begin{tabular}{|c|c|c|c|}
\hline & & & \\
\hline & Factors and FHIDS & Current & Normative \\
\hline I. & Trust vs Defensiveness (T) & & \\
\hline & 1. Lack of Cynicism & .68 & .68 \\
\hline & 2. Lack of Defensiveness & .43 & .59 \\
\hline & 3. Belief in Human Worth & .51 & .57 \\
\hline & 4. Trust in Human Nature & .62 & .66 \\
\hline & 5. Lack of Paranoia & .72 & .63 \\
\hline II. & Orderliness vs Lack of Compulsion (O) & & \\
\hline & 6. Neatness & .45 & .57 \\
\hline & 7. Routine & .52 & .59 \\
\hline & 8. Order & $.5 \%$ & .69 \\
\hline & 9. Caut iousness & .41 & .35 \\
\hline & 10. Meticulousness & .36 & .54 \\
\hline $\mathbb{I V}$. & Activity vs Lack of Energy (A) & & \\
\hline & 16. Fuercise & .45 & .51 \\
\hline & 17. Energy & .46 & .67 \\
\hline & 18. Need to Excel & .56 & .38 \\
\hline & 19. Liking for Work & .72 & .51 \\
\hline & 20. Stgmina & .68 & .70 \\
\hline V. & Fotional Stability vs Neuroticism(S) & & \\
\hline & 21. Lack of Inferiority Feelings & .49 & .59 \\
\hline & 22. Lack of Depression & .46 & .67 \\
\hline & 23. Lack of Agitation & .44 & .66 \\
\hline & 24. Lack of pessimism & .26 & .61 \\
\hline & 25. Mood Stability & .58 & .57 \\
\hline VI. & Extraversion vs Introversion (E) & & \\
\hline & 26. Lack of Reserve & .69 & .68 \\
\hline & 27. Lack of Seclusiveness & .59 & .60 \\
\hline & 28. No loss for Words & .72 & .84 \\
\hline & 29. Lack of Shyness & .75 & .79 \\
\hline & 30. No Stage Fright & .50 & .49 \\
\hline VII. I & Masculinity ys Femininity (M) & & \\
\hline & 31. No Fear of Bugs & .51 & .61 \\
\hline & 32. No Crying & .59 & .60 \\
\hline & 33. No Romantic Love & .16 & .43 \\
\hline & 34. Tolerance of Blood & .40 & .43 \\
\hline & 35. Tolerance of Vulgarity & .24 & .34 \\
\hline$V \amalg I I$. & Empathy VS Egocentrism $(\mathbb{P})$ & & \\
\hline & 36. Sympathy & .41 & .66 \\
\hline & 37. Helpfulness & .54 & .77 \\
\hline & 38. Service & .45 & .66 \\
\hline & 39. Generosity & .77 & .67 \\
\hline & 40. Unsel fishness & .53 & .57 \\
\hline
\end{tabular}


proval of society, and respect the law. As mentioned above, this factor was not separately identified in the current study as one of the seven obtained factors; hence, this factor has not been confirmed. Each of its EHIDs showed a major loading on one of the obtained factors, with two of them loading on the Orderliness vs. Lack of Compulsion (O) factor.

Activity vs. Lack of Energy (A). High-scoring individuals on this factor (Factor IV) have a great deal of energy and endurance, work hard, and strive to excel. The only FHID with a major loading that was not expected to define this factor was Meticulousness (No. 10), .38. The corresponding loading for this FHID in the normative analysis was .25. This FHD was supposed to define the Social Conformity vs. Rebelliousness (C) factor, which did not appear in the current analysis. Although the loadings for some FHIDs in the two analyses varied substantially (see Table 1), the overall pattern is sufficiently close to warrant the conclusion that Activity ws. Lack of Energy $(A)$ was confirmed in the current study.

Emotional Stability ws. Neuroticism (S). High-scoring individuals are relatively free of depression, optimistic, relaxed, stable in mood, and confident. Several FHIDs not expected to define this factor had loadings of .3 or more in the current study: Belief in Human Worth (No. 3), .36; Cautiousness (No. 9), .39; Law Enforcement (No. 11), .43; Respect for Law (No. 14), .47; Energy (No. 17), .34; Stamina (No. 20), .36; and No Loss for Words (No. 28), .33. None of the corresponding loadings for these FHIDs on this factor in the normative sample (Factor $V$ ) was larger than .3. The two largest unexpected loadings here were for EHID variables 11 and 14 , which were expected to define the Social Conformity vs. Rebelliousness (C) factor, not separately identified in the current study. The identification of this factor in the current study is clear (see Table 1), although the magnitudes of the loadings was smaller here than it has usually been in other samples.
Extraversion vs. Introversion (E). Persons with high scores on this factor (Factor VI) meet people easily, seek new friends, feel comfortable with strangers, and do not suffer from stage fright. Two FHIDs not expected to define this factor had loadings of .3 or more in the current analysis: Energy (No. 17), .35; and Need to Excel (No. 18), 35. The corresponding loadings in the normative sample were .31 and .14 , respectively. The degree of agreement between the current and past results (see Table 1) with respect to Extraversion vs. Introversion (E) could hardly be better. This was not only the strongest factor in the current study but also the best confirmed of the factors in the taxonomy in the current resullts.

Masculinity is. Femininity (M). High-scoring individuals tend to be tough minded; not bothered by blood, crawling creatures, or vulgarity; and do not cry easily or show much interest in love stories. Loadings of . 3 or more for FHIDs not expected to define this factor (Factor VII) were as follows: Lack of Defensiveness (No. 2), .35; Lack of Agitation (No. 23), .37, and Sympathy (No. 36), -.37. The corresponding loadings in the normative analysis were $-.05, .18$, and -.26 , respectively. This was the smallest of the seven factors obtained in the current analysis in terms of sum of squares of loadings; but the agreement with past results is very good (see Table 1), despite the fact that the original factor was not as well defined as other factors in the taxonomy. Loadings in the normative data for FHID variables 33,34 , and 35 were not as high as is desirable for defining variables. Although this factor is not as clear-cut and homogeneous as the other factors in the taxonomy, it has proved to be very useful in climical interpretation (Comrey, 1980).

Empathy ws. Egocentrism (P). High-scoring individuals describe themselves as helpful, generous, sympathetic people who are interested in devoting their lives to the service of others. There was no loading of .3 or more in the current analysis for any FHID not expected to de- 
fine this factor (Factor VIII). Although the loadings are somewhat lower for most FHIDs in the current analysis (see Table 1), the agreement of the present factor with the corresponding factor in the original normative study is sufficient to leave no doubt that this factor has been confirmed.

\section{Discursiman}

The results presented above demonstrate a substantial confirmation of seven of the eight personality factors from the taxonomy upon which the Comrey Personality Scales were constructed. Some of the factors were clearly more identical to the originals than others, but the overall level of agreement is substantial. This is especially true when the following factors, which could have been expected to make it difficult to achieve agreement are considered:

1. A translation of the original test to a new language was used to obtain the data:

2. Subjects were drawn from an entirely differ. ent culture and nation;

3. Subjects were taken from the middle and lower classes rather than from the middle and upper middle classes, as in the American sample;

4. Subjects were taking the test to obtain licensing rather than as volunteers seeking information only for their own private purposes;

5. The number of legitimate factors retained was determined by the data characteristics rather than being imposed by the fact that eight factors were expected;
6. The rotational solution was determined by the normal varimax method (Kaiser, 1958) rather than being a procrustean solution designed to force maximum conformity with previous results.

It is not clear why Social Conformity vs. Rebelliousness $(C)$ failed to emerge as a strong separate factor in the Israeli data. This factor has always been somewhat difficult to differentiate from Orderliness vs. Lack of Compulsion $(O)$ because the two factors typically correlate rather highly, even though an orthogonal factor solution is being forced on the data for purposes of convenience. In this study, the fact that testing took place in a government institution for purposes of licensing may have discouraged the expression of anti-establishment sentiment, thereby reducing the variance and the intercorrelations of FHDs that elsewhere identify this factor. Some evidence for the validity of this interpretation is given in Table 2, which shows that the means of the Social Conformity vs. Rebelliousness (C) FHIDs were substantially higher for the Israeli sample than for the United States normative sample, and the standard deviations were lower in most instances. The correlations among the FHIDs are also much lower in the Israeli sample (see Table 3). What effect other considerations, such as the translation of the items, may have had in causing this factor not to appear in the Israeli data is unknown at the present time.

The net effect of this study is to suggest a substantial robustness for the factor analytic personality taxonomy underlying the Comrey Personality Scales across cultural and language

Table 2

Social Conformity vs Rebelliousness (C) FHID Data

\begin{tabular}{|c|c|c|c|c|c|}
\hline \multirow{2}{*}{\multicolumn{2}{|c|}{ FHID }} & \multicolumn{2}{|c|}{ USA Sample } & \multicolumn{2}{|c|}{ Israel I Sample } \\
\hline & & Nean & S.D. & Mean & S.D. \\
\hline 11. & Larw Enforcement & 14.6 & 4.63 & 19.0 & 3.50 \\
\hline 12 。 & Acceptance of Social Order & 13.0 & 4.74 & 17.2 & $\$ .92$ \\
\hline 13 . & Intolerance of Non-Conformity & 12.3 & 4.82 & 18.5 & 4.42 \\
\hline 14. & Respect for llaw & 19.2 & 5.32 & 23.2 & 3.55 \\
\hline 15. & Need for Approval & 16.2 & 4.79 & 20.6 & 4.94 \\
\hline
\end{tabular}


Table 3

Intercorrelations Among Social Confomity vs Rebelliousness (C) FHIDS in the United States (Below Diagonal) and Istrael (Above Diagonal)

\begin{tabular}{lrrrrr}
\hline FHID & 11 & 12 & 13 & 14 & 15 \\
\hline 11. Law Enforcenent & & .03 & .06 & .27 & .20 \\
12. Acceptance of Social Order & .61 & & .22 & .10 & .17 \\
13. Intolerance of Non Confomity & .64 & .50 & & .02 & .32 \\
14. Respect for Law & .58 & .52 & .56 & & .15 \\
15. Need for Approval & .34 & .33 & .40 & .36 & \\
\hline
\end{tabular}

barriers. The results obtained lend credence to the view that these variables can be as useful for psychological screening and research in Israel as they have been found to be in the United States and other English-speaking countries.

\section{Defereres}

Comrey, A. L. EdITS manual for the Comrey Pepsonality Scales. San Diego CA: Educational and Industrial Testing Service, 1970.

Comrey, A. L. A first course in factor analysis. New York: Academic Press, 1973.

Comrey, A. L. Handbook of interpretations for the Comrey Personality Scales. San Diego CA: Educational and Industrial Testing Service, 1980.

Comrey, A. L., \& Backer, T. E. Detection of faking on the Comrey Personality Scales. Multivariate Behavioral Research, 1975, 10, 311-320.

Eysenck, H. I., \& Eysenck, S. B. G. Psychoticism as a dimension of personality. London: Hodder \& Stoughton, 1976.

Eysenck, S. B. G., Eysenck, H.I., \& Show, L. The modification of personality and lie scale scores by special "honesty" instructions. Britsh Joumal of Social and Clinical Psychology, 1974, 13, 41-50.

Forbes, A.R. Dexter, W. R., \& Comrey, A. L. A cross-cultural comparison of certain personality factors. Multivariate Behavioral Research, 1974, 9, 383-394.

Kaiser, H. F. The varimax criterion for analytic rota tion in factor analysis. Psychometrika, 1958, 23, $187-200$

Montag, I. Adequate response set and social desirability in personality guestionnaires. Unpublished doctoral dissertation, Tel Aviv University, 1978.

Rodrigues, A., \& Comrey, A. L. Personality structure in Brazil and the United States. Joumal of Social Psychology, 1974, 92, 19-26.

Vandenberg, S. G., \& Price, R. A. Replication of the factor structure of the Comrey Personality Scales. Psychological Reports, 1978, 42, 343-352.

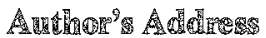

Send requests for reprints or further information to Andrew L. Comrey, Department of $\mathbb{E}$ sychology, University of Califormia, Los Angeles CA 90024. 